\title{
Cluster organization of miRNA binding sites in mRNA of atherosclerosis candidate genes
}

\author{
Dina Mukushkina \\ SRI of biology and biotechnology \\ problems \\ Al-Farabi Kazakh National University \\ Almaty, Kazakhstan \\ dina.mukushkina@gmail.com
}

\author{
Dana Aisina \\ SRI of biology and biotechnology \\ problems \\ Al-Farabi Kazakh National University \\ Almaty, Kazakhstan \\ dana.aisina03@gmail.com
}

\author{
Anatoliy Timofeevich Ivashchenko \\ SRI of biology and biotechnology \\ problems \\ Al-Farabi Kazakh National University \\ Almaty, Kazakhstan \\ a.iavashchenko@gmail.com
}

\begin{abstract}
Many candidate genes are involved in the development of atherosclerosis, which are manifested in a variety of metabolic processes, disturbances of which are observed in this disease. Many publications describe the involvement of miRNAs in the development of atherosclerosis. Therefore, it is necessary to establish which miRNAs can affect the expression of candidate genes. Quantitative characteristics of the interaction of miRNAs with mRNAs candidate genes were determined using the MirTarget program, which detects localization of miRNA binding sites in 3'UTR, 5'UTR and CDS; free energy interaction miRNA with mRNA; schemes of nucleotide interaction between miRNA and mRNA. In the mRNA of atherosclerosis genes, the binding sites of two or more miRNAs located in 3'UTR, 5'UTR and CDS are established. The organization of miRNAs binding sites with overlapping nucleotide sequences forming clusters was revealed. Such an organization of miRNAs binding sites leads to their compaction several times and causes competition among miRNAs for binding to mRNA. The features of the interaction of miRNAs with mRNA of candidate genes depending on their expression are established. Associations of miRNAs and candidate atherosclerosis genes are proposed for the early diagnosis of this disease.
\end{abstract}

Keywords - miRNA, mRNA, gene, association, marker, atherosclerosis

\section{INTRODUCTION}

Candidate genes are participated in the development of atherosclerosis, which are implicated in a variety of metabolic processes that are disturbed in this disease. It has been found that miRNAs, which are nanoscale regulatory biomolecules, are involved in many biological processes at all stages of the development of atherosclerosis, from early endothelial dysfunction to the erosion and rupture of an unstable atherosclerotic plaque. The miRNAs are able to regulate gene expression at the levels of translation binding to target mRNAs. Many publications have described the involvement of miRNAs in the development of atherosclerosis. It is necessary to establish which miRNAs affect the expression of candidate genes.

\section{METHODS AND ALGORITHMS}

The nucleotide sequences of 2565 miRNAs were downloaded from the miRBase (http://mirbase.org, Release 22.1) and 3707 miRNAs obtained from a report by Londin E. et al. [1]. The nucleotide sequences of mRNA genes were obtained from GenBank (http://www.ncbi.nlm.nih.gov). A database of 91 candidate genes including the names of the genes and publication sources (PubMed database) was compiled, confirming the associations of these genes with atherosclerosis. A search for the target genes of miRNAs was performed using the MirTarget program [2]. This program determines the following binding characteristics: the start of the miRNA-binding site of mRNA; the locations of miRNA-binding sites (3'UTR, 5'UTR, CDS); the interaction free energy $(\Delta \mathrm{G}, \mathrm{kJ} / \mathrm{mole})$; nucleotide interaction schemes between miRNAs and mRNAs.

\section{RESULT}

Binding sites for 24 mRNAs genes with miRNAs in the 5'UTR region were identified. The binding sites of the miRNAs and mRNAs of the target genes were not uniform along the length of an mRNA. Both multiple and single binding sites were identified. The mRNA regions containing overlapping binding sites are referred to clusters [3]. The results regarding the location of miRNA-binding sites in mRNAs with overlapping nucleotide sequences in a cluster leads to the compaction and causes competition among miRNAs. The mRNA of GAS6 gene forms a large cluster with 22 different miRNAs, half of which form two or three binding sites, while the rest represent single binding sites. The cluster size is 37 nucleotides. The total binding sites length in cluster is 858 nucleotides, where the degree of compaction is 23.2. This compaction allowed these miRNA binding sites to be located in 5'UTR with a length of 153 nucleotides. For eight associations of miRNAs with mRNA GAS6 gene the free interaction energy of miRNAs with mRNA have been more than $-130 \mathrm{~kJ} /$ mole, which allows us to recommend these associations as markers of atherosclerosis. The cluster of binding sites of 18 miRNAs в the NFE2L2 gene 5'UTR.mRNA have size of 45 nucleotides (Table). The total size of all binding sites is 521 nucleotides, which means that the degree of compaction is 11.6. Nine miRNAs interacted with the mRNA of the NFE2L2 gene with a free energy of more than $-130 \mathrm{~kJ} / \mathrm{mole}$ are recommended as markers of atherosclerosis. The 41 genes exhibited one binding site in CDS mRNAs for one miRNA. In CDS of IRS 2 mRNA the binding sites of 28 miRNAs were located in cluster with a length of 41 nucleotides. The total cluster size was 1147 nucleotides, while the degree of cluster compaction was 28.0. The binding sites of 12 miRNAs were located in other cluster with a length of 35 nucleotides. The PDE $4 D$ gene forms two clusters but smaller. 


\begin{tabular}{|c|c|c|c|c|c|c|}
\hline Gene & miRNA & $\begin{array}{c}\text { Region of } \\
\text { RNA }\end{array}$ & $\begin{array}{c}\text { Start } \\
\text { of site, } n t\end{array}$ & $\begin{array}{c}\Delta \mathrm{G}, \\
\mathrm{kJ} / \mathrm{mole}\end{array}$ & $\begin{array}{c}\Delta \mathrm{G} / \Delta \mathrm{Gm}, \\
\%\end{array}$ & $\begin{array}{c}\text { Length, } \\
\text { nt }\end{array}$ \\
\hline \multirow[t]{13}{*}{ NFE2L2 } & ID00522.5p-miR & 5'UTR & 438 & -125 & 89 & 23 \\
\hline & ID02187.5p-miR & 5'UTR & $439 \div 445(2)$ & -123 & 89 & 23 \\
\hline & ID03367.5p-miR & 5'UTR & $441 \div 453(2)$ & -115 & 92 & 20 \\
\hline & ID01804.3p-miR & 5'UTR & $442 \div 444(2)$ & -132 & 90 & 23 \\
\hline & ID02294.5p-miR & 5'UTR & 443 & -132 & 90 & 24 \\
\hline & ID00061.3p-miR & 5'UTR & $444 \div 450(3)$ & $-125 \div-134$ & $91 \div 97$ & 22 \\
\hline & ID00457.3p-miR & 5'UTR & 444 & -123 & 91 & 22 \\
\hline & ID01041.5p-miR & 5'UTR & $444 \div 445(2)$ & $-129 \div-134$ & $88 \div 91$ & 24 \\
\hline & ID01873.3p-miR & 5'UTR & $444 \div 447(2)$ & $-121 \div-123$ & $92 \div 94$ & 21 \\
\hline & ID00296.3p-miR & 5'UTR & 448 & -140 & 89 & 25 \\
\hline & ID01641.3p-miR & 5'UTR & 448 & -136 & 91 & 24 \\
\hline & ID01702.3p-miR & 5'UTR & 448 & -138 & 92 & 24 \\
\hline & ID02890.3p-miR & 5'UTR & 458 & -119 & 89 & 23 \\
\hline \multirow[t]{12}{*}{$P D E 4 D$} & ID00061.3p-miR & $\mathrm{CDS}$ & $391 \div 413(3)$ & $-125 \div-129$ & $91 \div 94$ & 22 \\
\hline & ID00457.3p-miR & CDS & 392 & -123 & 91 & 22 \\
\hline & ID01315.3p-miR & $\mathrm{CDS}$ & 392 & -115 & 92 & 20 \\
\hline & ID01377.3p-miR & CDS & 394 & -121 & 95 & 20 \\
\hline & ID01705.3p-miR & $\mathrm{CDS}$ & 398 & -117 & 92 & 21 \\
\hline & ID00296.3p-miR & CDS & $404 \div 411(4)$ & $-140 \div-142$ & $89 \div 91$ & 25 \\
\hline & ID01641.3p-miR & CDS & $407 \div 410(2)$ & $-132 \div-132$ & 89 & 24 \\
\hline & miR-3960 & CDS & $408 \div 415$ & $-115 \div-117$ & $92 \div 93$ & 20 \\
\hline & ID01458.5p-miR & $\mathrm{CDS}$ & 410 & -134 & 91 & 23 \\
\hline & ID01702.3p-miR & CDS & 411 & -138 & 92 & 24 \\
\hline & ID02064.5p-miR & CDS & 415 & -129 & 90 & 23 \\
\hline & ID01184.3p-miR & CDS & 419 & -117 & 93 & 20 \\
\hline
\end{tabular}

What is characteristic is that miRNA ID01641.3p-miR, ID00061.3p-miR, miR-3960 and ID01702.3p-miR are found in both clusters. The first cluster consists of four single binding sites and one multiple binding site ID01702.3p-miR, cluster size is 35 nucleotides, from the position of 335 nucleotides and to 369 nucleotides. The average $\Delta \mathrm{G}$ value is $-130 \mathrm{~kJ} / \mathrm{mole}$. The second cluster consists of four multiple binding sites and six single binding sites, the cluster size is 49 nucleotides from 391 to 439 nucleotides (Table). The total cluster size is 447 nucleotides and degree of compaction was 9.1 .

Clusters consisting of single miRNA-binding sites were found in the mRNA following genes: ACE, ADAMTS13, $A D R B 3, I C A M 1$ and FASLG. In 3'UTR mRNA of 45 genes were characterized binding sites. In $A D R B 3, C D 36, F A S L G$, F11R, FLT1, ICAM1, PLA2G7, PPARGC1A mRNAs the clusters of miR-466, ID00436.3p-miR, ID01030.3p-miR binding sites were established. The binding of 53 miRNAs with mRNAs of 14 candidate genes with free energy interaction more than $-130 \mathrm{~kJ} / \mathrm{mole}$ were established. The miR-619-5p was fully complementary to $A D A M 17$ and CD36 mRNA genes, ID01593.5p-miR to ANGPTL4 mRNA gene, ID01935.5p-miR to NFE2L2 gene and miR-5096 to IL 18 mRNA gene.

\section{CONCLUSION}

The miRNA associations with atherosclerosis candidate genes have been established, which consist of: one gene and several miRNAs; one gene and one miRNA; one miRNA and several genes; two or more miRNAs with two or more candidate genes. sites in mRNA candidate genes contributesto a more accurate diagnosis of the participation of competing miRNAs in the development of atherosclerosis.

\section{ACKNOWLEDGEMENTS}

The work was carried out with the financial support of the Ministry of Education and Science of the Republic of Kazakhstan within the framework of the grant №AP05132460. We are grateful to Pyrkova A.Yu. to performing calculations on the program MirTarget.

\section{REFERENCES}

[1] E. Londin, et al. "Analysis of 13 cell types reveals evidence for the expression of numerous novel primate- and tissue-specific microRNAs," PNAS, pp. E1106-E1115, 2015.

[2] A. T. Ivashchenko, A. Y. Pyrkova, R. Y. Niyazova, A. Alybayeva, $\mathrm{K}$. Baskakov, "Prediction of miRNA binding sites in mRNA," Bioinformation, vol. 12. pp. 237-240. 2016.

[3] D. Aisina, R .Niyazova, S. Atambayeva, A. Ivashchenko, "Prediction of clusters of miRNA binding sites in mRNA candidate genes of breast cancer subtypes," PeerJ. vol. 7. pp. e8049, 2019. doi: 10.7717/peerj.8049 